\title{
Role of host star variability in the detectability of planetary phase curves
}

\author{
D. Hidalgo ${ }^{1,2}$, R. Alonso ${ }^{1,2}$, and E. Pallé ${ }^{1,2}$ \\ ${ }^{1}$ Instituto de Astrofísica de Canarias, 38205 La Laguna, Tenerife, Spain \\ e-mail: dhidalgo@iac.es \\ 2 Departamento de Astrofísica, Universidad de La Laguna, 38206 La Laguna, Tenerife, Spain
}

Received 31 August 2018 / Accepted 17 October 2018

\begin{abstract}
Phase curves, or the change in observed illumination of the planet as it orbits around its host star, help us to characterize their atmospheres. However, the variability of the host star can make their detection challenging. The presence of starspots, faculae, flares, and rotational effects introduce brightness variations that can hide other flux variations related to the presence of an exoplanet: ellipsoidal variation, Doppler boosting, and a combination of reflected light and thermal emission from the planet. Here we present a study to quantify the effect of stellar variability on the detectability of phase curves in the optical. In the first stage we simulated ideal data, with different white noise levels, and with cadences and total duration matching a quarter of the Kepler mission. We performed injection and recovery tests to evaluate the minimum number of planetary orbits that need to be observed in order to determine the amplitude of the phase curve with an accuracy of $15 \%$. We also evaluate the effect of a simplistic stellar variability signal with low amplitude in order to provide strong constraints on the minimum number of orbits needed under these ideal conditions. In the second stage we applied these methods to data from Q9 of the Kepler mission, known for its low instrumental noise. The injection and recovery tests are performed on a selected sample of the less noisy stars in different effective temperature ranges. Even for the shortest explored planet period of 1 day, we find that observing a single orbit of the planet fails to detect accurately more than $90 \%$ of the inserted amplitude. The best recovery rates, close to $48 \%$, are obtained after 10 orbits of a 1 day period planet with the largest explored amplitude of $150 \mathrm{ppm}$. The temperature range of the host stars providing better recovery ratios is $5500 \mathrm{~K}<T_{\text {eff }}<6000 \mathrm{~K}$. Our results provide guidelines to selecting the best targets in which phase curves can be measured to the greatest accuracy, given the variability and effective temperature of its host star, which is of interest for the upcoming TESS, CHEOPS, and PLATO space missions.
\end{abstract}

Key words. planets and satellites: gaseous planets - planets and satellites: atmospheres - planets and satellites: detection stars: activity - techniques: photometric

\section{Introduction}

Studying planetary systems which transit their host star provides a great opportunity to derive their physical properties. During a transit we can measure the exoplanet radius $\left(R_{p}\right)$ in units of the stellar radius $\left(R_{*}\right)$ through the depth of the transit $\sim\left(R_{p} / R_{*}\right)^{2}$, the scaled semi-major axis, the impact parameter (Mandel \& Agol 2002), and the orbital period. If the geometry (inclination and eccentricity) of the system is adequate, a secondary eclipse can also be observed. Along one orbital period, the time-dependent change in the brightness of the light curve measured due to the reprocessed light of the exoplanet from its host star is known as the phase curve. This change in brightness is determined by the combination of emitted and reflected light from the host star in a particular bandpass (Cowan \& Agol 2011).

Secondary eclipses and phase curves can provide a wealth of information on a given planet and help characterize its atmosphere. The depth of the secondary eclipses gives us clues to constrain the albedo of the planet (Angerhausen et al. 2015), while the timing and duration help us to determine the orbital parameters (Agol \& Fabrycky 2017). A detailed study of phase curve can tell us, for instance, how efficient the energy redistribution is between the exoplanet's dayside and nightside, or how atmospheric bright spots can shift due to persistent wind patterns (e.g. Knutson et al. 2007; Demory et al. 2013; Armstrong et al.
2016). All these effects can be characterized in different wavelengths in order to show the vertical temperature structure and also the chemical composition of the exoplanet atmosphere.

Phase curves have been observed in the near- and midinfrared using the Spitzer Space Telescope for several planets, such as HD 209458b (Zellem et al. 2014), the hot Saturn HD 149026b (Knutson et al. 2009) or WASP-43b (Stevenson et al. 2017). With the launch of Kepler Space Telescope (Borucki et al. 2008), phase curves have also been observed in the optical (e.g. Kepler-7b; Esteves et al. 2013). Only one phase curve in the optical band had been previously observed: CoRoT-1b (Snellen et al. 2009). In the optical band, the planet-star contrast is much lower, and the contributions from ellipsoidal variations or the beaming effect (also named Doppler boosting) become important (e.g. Loeb \& Gaudi 2003; Zucker et al. 2007; Shporer 2017). It should also be noted that in this bandpass, the phase curve is degenerated. In the extreme cases of hot Jupiters, there is a component of the planet's own emission that contaminates the signal, leaking into the Kepler bandpass (e.g. López-Morales \& Seager 2007; Heng \& Demory 2013). For further insight into observations and theory of phase curves see Parmentier \& Crossfield (2017).

High-precision space photometry is a powerful tool for the study of transits and secondary eclipses as well as phase curves. However, aside from the systematic variations of photometry due to telescope movement and other electronic processing, the 
Table 1. Examples of typical amplitudes of phase curves measured in Kepler data (from Esteves et al. 2013).

\begin{tabular}{cc}
\hline \hline & Amplitude ppm \\
\hline Kepler 5b & $19.3_{-5.3}^{+6.3}$ \\
Kepler 7b & $48 \pm 13$ \\
Kepler 12b & $22.9_{-4.1}^{+4.3}$ \\
Kepler 43b & $71 \pm 44$ \\
Kepler 76b & $106.9_{-4.4}^{+4.3}$ \\
KOI-13b & $150.4 \pm 2.7$ \\
HAT-P-7b & $73.3^{ \pm 2.7}$ \\
TrES-2b & $4.1_{-1.0}^{+1.1}$ \\
\hline
\end{tabular}

intrinsic variability of the star itself plays a fundamental role when performing data reduction and analysis.

The Sun has been studied in depth to unravel the mechanisms of stellar activity emerging from the surface (Berdyugina 2005) and this activity can also be seen in other stars. Dark starspots corresponding to concentrations of the magnetic field emerging from the photosphere, while bright faculae correspond to the enhanced network magnetic field, which is dispersed over a much larger area (Kitiashvili et al. 2013). In general, stellar variability usually shows periodicity. However, in many cases, this periodicity may vary because the configuration of the magnetic field on the star's surface changes rapidly over time and results in irregular variability in the light curve (Brown \& Gilliland 1994).

Serrano et al. (2018) have published a study focusing on the distinction of exoplanet albedo from stellar activity. Unlike their study which focuses on the fit of stellar variability to obtain an albedo estimation, requiring a continuous time interval of data for at least one stellar rotation, the study we present here focuses on fitting the phase curve without the need for these phase curves to be consecutive. This is the case when the scheduling of long observations is not easily possible (e.g. repetition and combination of HST phase curves, K2 re-observations of the same star) or observations that are part of a tight schedule of different programs (e.g. CHEOPS or JWST).

For the new generation of space-based telescopes such as TESS (Ricker et al. 2015), CHEOPS (Broeg et al. 2013) and PLATO (Rauer et al. 2014), it is important to determine the phase curve detection limitations due to stellar variability in order to prepare the exploitation of their data.

In this paper, we determine the detectability of optical phase curves of exoplanets using the Kepler database (Jenkins et al. 2010). In Sect. 2, we perform simulated phase curve injection tests and describe the analysis procedure. In Sect. 3, we apply the same methodology to Kepler data. We distinguish between two cases: one where we attempt to filter out the stellar variability and another where we do not, since in many cases we do not have enough information to perform the filtering. Finally, in Sect. 4, we discuss the results and present our conclusions.

\section{Phase-curve injection tests on simulated data}

Throughout this manuscript, we define one orbit as the entire temporal interval that goes from the exit of a primary transit to the beginning of the next transit. As the goal is to study the influence of stellar variability that cannot be corrected, we adopt several ideal assumptions that explore the minimum effect of this variability:

- The phase curve is injected and modeled by a simple sinusoidal. Higher harmonics such as tidal modulations are not included in this study.
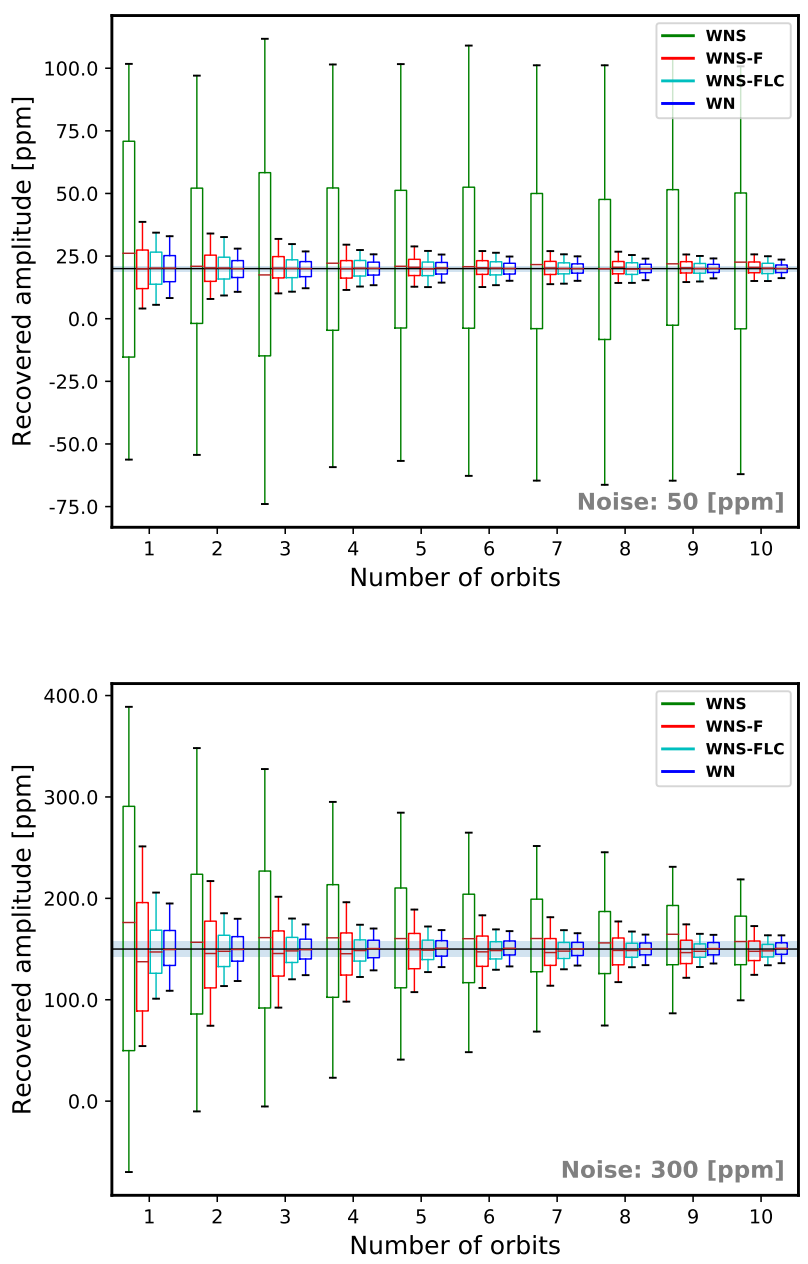

Fig. 1. Results of the recovery of the amplitude of phase curves versus the number of orbits with which the fit has been made. Each plot represents an example light curve generated with different white noise and amplitude (top panel: noise of $50 \mathrm{ppm}$ per exposure, amplitude of $20 \mathrm{ppm}$, and period of 2.5 days; bottom panel: noise $300 \mathrm{ppm}$ per exposure, amplitude of $150 \mathrm{ppm}$, and period of 7.5 days) for an exposure time of $29.4 \mathrm{~min}$. The horizontal black line indicates the amplitude of the entered phase curve and the shaded area in blue is the range in which the phase curve detection criterion is valid. For each combination number of orbits, there are four boxplots that indicate the four different cases considered: WN (blue), WNS (green), WNS-F (red), and WNS-FLC (light blue-green). The red horizontal line on each boxplot indicates the median, the rectangular box is the IQR (see definition in Sect. 2.3) and the thin error bars span the whole distribution of points. In the top figure there is no detection, as the IQR is not fully inside the blue-shaded area, whereas in the bottom figure there is a detection when we accumulate seven or more orbits.

- The period and orbital phase are exactly known, and we will only study the effects on the amplitude of the sinusoid.

Any departures from the previous assumptions will increase the detrimental effects of the stellar activity on the phase curve determinations. In our study, the amplitude of the phase curve is defined as the peak to peak difference. Our injected light curves are sinusoidal signals shifted by $\pi / 2$ (so that the maximum is in phase 0.5 ). The amplitude of the phase curve is thus twice the sinusoidal semi-amplitude.

\subsection{White noise injection}

Since we systematically introduced simulated phase curves in the form of sinusoidal signals into Kepler light curves (see 

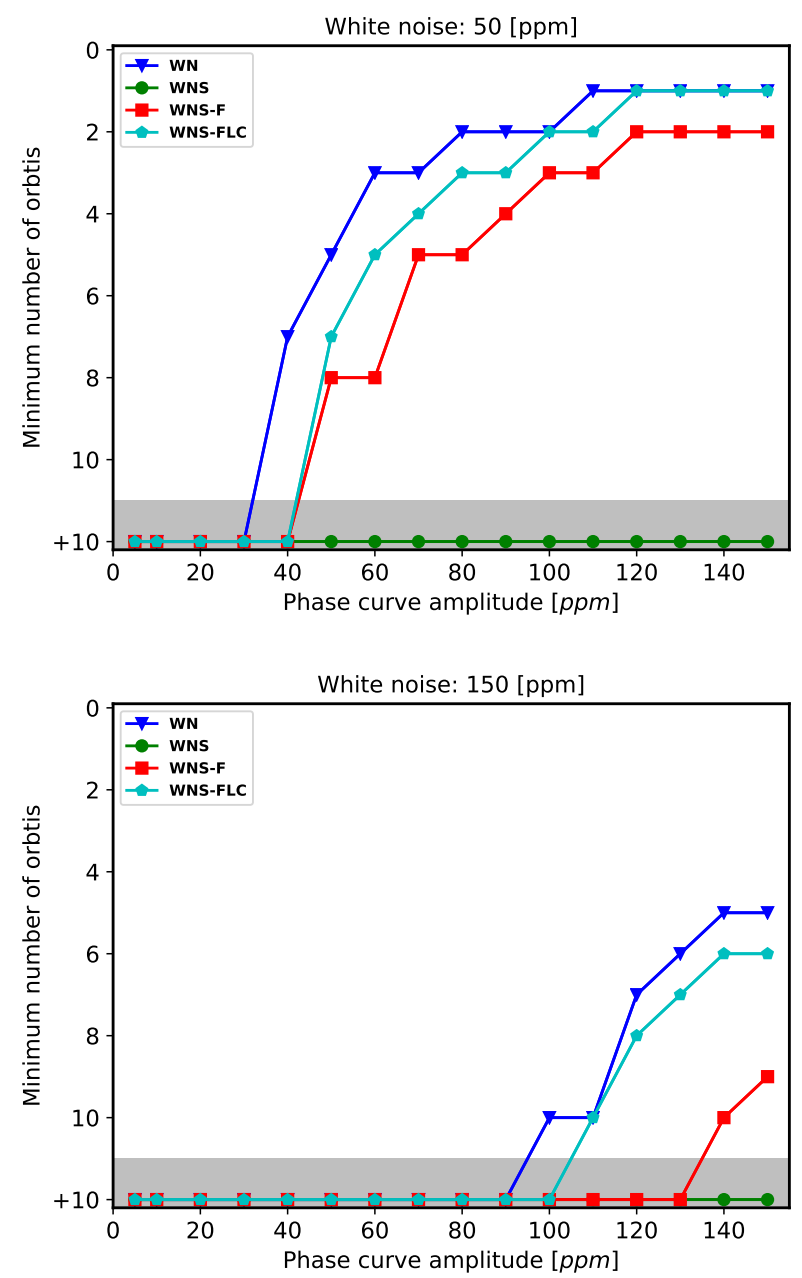

Fig. 2. Minimum number of orbits required to have significant phase curve detection. Each of the plots represents an initial white noise level (top panel: $50 \mathrm{ppm}$, bottom panel: $150 \mathrm{ppm}$ ) for a period of 2.5 days. For each of the different white noise curves, four different cases are studied, as explained in Fig. 1. The grey shaded area represents the case where more than 10 orbits $(+10)$ are required to perform a phase curve detection. The plot with an amplitude of $300 \mathrm{ppm}$ is not presented here since it is necessary more than 10 orbits in all cases to detect the phase curve.

next section), we first used simulated light curves to check that the method would give consistent results. We decided to use Kepler's long cadence (LC) time steps of 29.4 min to unify criteria with the data used in Sect 3. For our mock light curves, we also choose a duration equal to the quarter 9 (Q9) of the original mission Kepler. With only one quarter we can perform our study, and the quarter 9 has been chosen because it has the lowest noise levels (Howell et al. 2016).

We generated white noise light curves with a normal distribution centred on 1.0, as if it were normalized, and with a standard deviation of 50,150, and $300 \mathrm{ppm}$ per exposure and for an exposure time of $29.4 \mathrm{~min}$. The width of the distribution was chosen taking into account the expected photometric performance of the next generation of space missions: TESS, CHEOPS, and PLATO. For the CHEOPS mission, we obtained this precision with magnitudes in the $V$-band of $\sim 9.6$, of slightly more than $\sim 12$, and of $\sim 13$, respectively, according to the exposure time calculator ${ }^{1}$ from the CHEOPS guest observers

1 https://www. cosmos. esa.int/web/cheops-guestobservers-programme/open-time-workshop-2017 programme. For TESS, these magnitudes correspond to $\sim 10$ and 8.5 for the case of 300 and $150 \mathrm{ppm}$, respectively ${ }^{2}$. The TESS telescope is not able to reach the estimated photometric error of $50 \mathrm{ppm}$ since the mission assumes $60 \mathrm{ppm}$ of background noise on hourly timescales (Ricker et al. 2015). PLATO is still under development, but a first estimation can be made (Rauer et al. 2016) and it corresponds approximately to $\sim 11, \sim 13.5$, and $\sim 15$, respectively.

\subsection{Stellar variability and phase curve injection}

Our next step was to introduce stellar variability into our mock light curves. The stellar variability in Kepler light curves has already been studied (see the histogram in Fig. 4 of Basri et al. 2011). In both cases, for those stars that present periodicity in their variability and for those that do not, the most frequent amplitude of variability is around 2000 ppm. Their Fig. 7 shows that the most frequent period for stars with variability around $2000 \mathrm{ppm}$ is $14-15$ days. If we take into consideration these results, a typical range for the amplitude of stellar variability is between 100 and $15 \times 10^{3} \mathrm{ppm}$ and periods between 8 and more than 14 days for that range, respectively. We assumed a relatively favourable scenario for the detection of phase curves by introducing stellar variability in our light curves through a sinusoidal signal with an amplitude of $500 \mathrm{ppm}$ and a period of 25.6 days (like the Sun).

We simulated an idealized effect of stellar variability by introducing a second sinusoidal signal with a specific period and amplitude. We introduced periods much shorter than the period of stellar variability, namely: 1.0, 2.5, 5.0 and 7.5 d. As for the amplitude of the phase curve, we explored the values: $5 \mathrm{ppm}$ and from 10 to $150 \mathrm{ppm}$ in steps of $10 \mathrm{ppm}$. These values span the typically detected amplitudes ranges for known exoplanets (see in Table 1).

\subsection{Light curve analysis}

Our mock light curves have a duration of 97 days, covering a significant amount of phase curves depending on the period we are using: $1.0,2.5,5.0$, or 7.5 days. Phase curves cannot always be continuously observed from space due to mission planning constraints. For example, CHEOPS can only observe phase curves individually.

For this reason, we combined subsample datasets of our mock light curves, covering up to a maximum of ten nonconsecutive, randomly chosen phase curves. The combination was done in order to increase the signal-to-noise ratio and to be able to better fit the simulated phase curve function. All fits were made using non-linear least-squares minimization.

While recovering the phase curves, we explored the following cases:

- WN: Light curves containing only white noise + the injected phase curve;

- WNS: Same as in the previous case, with the added effect of stellar variability, but without filtering that variability;

- WNS-F: Same as before, but filtering stellar variability locally. This local filtering consists of taking each orbit individually and normalizing it by the median. In addition, since orbits can be found anywhere on the curve, we eliminate the local slope of an orbit by fitting a straight line;

- WNS-FLC: light curves again containing stellar variability, and here with a detrending filter applied to the whole light

2 https://heasarc.gsfc.nasa.gov/cgi-bin/tess/webtess/ wtm.py 


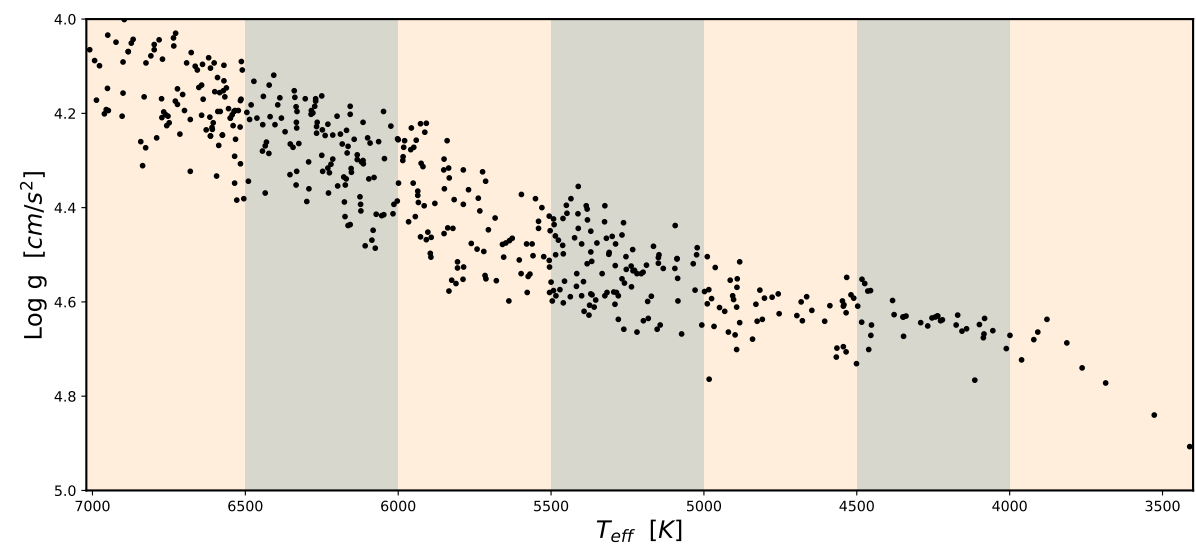

Fig. 3. Logarithm of gravity versus effective temperature for the entire sample of stars used in this study, taken from Kepler Input Catalog (KIC; Brown et al. 2011). The different shaded zones correspond to the effective temperature ranges in which the sample has been subdivided. curve to try to remove it. This filter consists of performing binning so each orbit is represented by a point, and then an interpolation fit function by third-order splines.

To retrieve the statistical significance of our study, we performed multiple combinations of different orbits in different parts of the light curve in order to construct a distribution of the results.

The combination of two or more phase curves gives rise to a large number of possibilities. For each of the cases (from 2 to $n$ orbit combinations, but we limit our study to 10 orbits), we made 100 random combinations of individual orbits. We also established a criterion to determine whether a phase curve is properly detected: when the distribution of amplitudes obtained with all orbit combinations has both its quartile 3 and quartile 1 completely included in the amplitude range from $-15 \%$ to $+15 \%$ of the injected amplitude, and this injected amplitude is in the interquartile range (IQR) of the distribution.

\subsection{Results of phase curves recovery}

We applied the method explained in the previous section to different curves generated with three levels of white noise, with standard deviations of 50, 150, and $300 \mathrm{ppm}$. A box plot analysis of independent light curves is shown in Fig. 1.

Following a similar analysis for all light curves, the final recovery results can be seen in Fig. 2, except for the 300 ppm case which did not produce any detection. The ideal case is the most favourable upper limit (blue line). On the other hand, the most realistic case, is where the light curve has stellar variability with no corrections, which is the lower limit (green line). In the middle (red and light blue line), are the results for different methods of detrending the stellar variability.

The recovery of the injected phase curve is possible in a relatively small number of orbits for low values of white noise or large phase curse semi-amplitudes, for the idealized case of no stellar variability, or when this stellar variability is corrected over the full light curve duration. When it is not possible to make a correction to this variability, no detection of any phase curve that we have introduced can be accomplished with less than 11 orbits (many more will be needed in most cases). This will be the situation for CHEOPS where the time interval of observation will not allow a trend in the variability of the star to be deduced.

In the case of $50 \mathrm{ppm}$ white noise assumption, we are able to detect phase curves with amplitudes greater than $60 \mathrm{ppm}$ for the WNS-FLC case or $70 \mathrm{ppm}$ for the WNS-F if we have at least five accumulated orbits with a period of 2.5 day. Conversely, for the $300 \mathrm{ppm}$ white noise assumption, we are not able to detect any phase curves with a period of 2.5 days with less than 10 orbits (not shown). In the case of $150 \mathrm{ppm}$ white noise, we are in an intermediate situation: we are able to detect amplitudes of the phase curve of more than $130 \mathrm{ppm}$ in the WNS-FLC case or $140 \mathrm{ppm}$ in the WNS-F case.

\section{Application to Kepler dataset}

In order to carry out this study with real photometric data, there is a minimum requirement: a time series long enough and a signal-to-noise ratio low enough to be able to detect the amplitude of the phase curve. The Kepler dataset is perfectly suited to these very specific requirements. Therefore, a number of stars from Kepler's complete star catalogue, taken directly from NASA Exoplanet Archive ${ }^{3}$, were selected to build the sample. The set of stars selected were downloaded from the database from the Mikulski Archive for Space Telescopes ${ }^{4}$ (MAST). For this study we used the light curves generated with the latest Kepler Data Release 25; more specifically, the flux vector used was created via simple aperture photometry (SAP) with a precise artefact mitigation included called Pre-search Data Conditioning (PDC_SAP; Smith et al. 2012).

Among all the stars in the Kepler catalogue, we rejected the red giants and we considered only main sequence stars with an effective temperature lower than $7000 \mathrm{~K}$ (where most hot Jupiters have been found) and with a Kepler magnitude brighter than 12 (to avoid instrumental noise on fainter targets). We divided the effective temperature range into different sub-ranges of $500 \mathrm{~K}$; in each sub-range we selected the 100 stars that have the smallest CDPP in $1.5 \mathrm{~h}$. The sub-ranges from 3500 to $5000 \mathrm{~K}$ contain 17, 37, and 48 stars, respectively.

We remove the giant stars by limiting the logarithm of gravity $(\log g)$. This limit varies from one temperature sub-range to another. Therefore, we take the greater $\log g$ value $\left(\max _{\log g}\right)$ for each temperature and manually remove all stars that have an approximate value above $\max _{\log g}-0.3$. In Fig. 3 we plot our sample of stars as a function of stellar effective temperature $T_{\text {eff }}$. Each shaded zone represents a sub-range in temperature.

To inject a planetary signal in our sample of selected Kepler light curves, we proceed as described in Sect. 2. Since these are real light curves, they already contain stellar variability signals and white noise. We extract the phase curve, using non-linear least-squares minimization, and use the same IQR criteria for detection.

\footnotetext{
3 https://exoplanetarchive.ipac.caltech.edu/

4 https://archive.stsci.edu/kepler/data_search/ search.php
} 
Figure 4 shows the fraction of phase curves with the correct amplitude recovered for an input period of 2.5 days. Equivalent plots for other input periods (1, 5, and 7.5) are shown in Appendix A. In each figure we show the combination of 1, 5, and 10 orbits, (top, middle, and bottom panels, respectively). As expected, the larger the amplitude of the injected phase curve, the greater the probability that the phase curve is recovered. The probability also increases as we accumulate more orbits. For a period of 2.5 days, Fig. 4 shows how the fraction of recovered phase curves is better as we accumulate orbits. In all cases, a phase curve is more easily detected when the star is of spectral type $G$ (solar type).

With one orbit, the fraction of phase curves is barely recovered, even for amplitudes larger than $130 \mathrm{ppm}$. Independent of the period and number of orbits, the best result is for sun-like stars (spectral type $G$, from 5500 to $6000 \mathrm{~K}$ ), but for one orbit the probability of recovering the phase curve is below $8 \%$. For the rest of the spectral types, the fraction of recovered phase curves is below $2 \%$ or null.

When five orbits are accumulated, the fraction of phase curves significantly increases. For $G$-type stars, the probability of recovery is below $35 \%$. For $G-F$ spectral types (yellow dotted line), this probability is around $20 \%$, and for the rest of the spectral types the recovery factor is below $10 \%$.

If we accumulate up to 10 orbits, the detection rate increases, but not significantly. For stars of $G$ spectral type, this probability reaches $45 \%$. For stars of $F-G$ type, the probability now is around $30 \%$, and for the rest of the spectral types it barely crosses the line of $10 \%$.

We performed the same type of study for the rest of the proposed periods (1.0, 5.0, and 7.5 days), obtaining similar results. In all cases, the behaviour is similar. The $G$ spectral type is the one with the best fraction of phase curve recovery. Independent of the inserted period, using only observations during one orbit is not enough to correctly obtain the amplitude of the phase curve in $8 \%$ of cases in the most favourable scenario. When $G$ stars have five orbits, a fraction of $\gtrsim 40 \%, \sim 28 \%$, and $\gtrsim 10 \%$ are obtained for a period of 1.0, 5.0, and 7.5 days, respectively. With 10 orbits, the behaviour is similar but with a higher fraction: $\gtrsim 50 \%, \sim 38 \%$, and $\gtrsim 30 \%$ for a period of $1.0,5.0$, and 7.5 days, respectively. For the rest of the spectral types, we find a behaviour similar to that shown in Fig. 4. The fraction of stars below $5500 \mathrm{~K}$ where the phase curve amplitude was correctly detected is much smaller, in most cases not reaching the barrier of $10 \%$. For stars warmer than $6000 \mathrm{~K}$, the pattern is similar. Having 10 accumulated orbits, there is a larger fraction of phase curve recovery, around $15 \%$ for periods below 5.0 days and $10 \%$ if the period is 7.5 days.

We note that the longer the period, the sooner the phase curves begin to be observed. For a period of 7.5 days and 10 accumulated orbits, the phase curve is recovered with an amplitude of $30 \mathrm{ppm}$. In the case of a 1.0 days period, the amplitude increases to $50 \mathrm{ppm}$.

\section{Summary and conclusions}

In this work, we studied the role of host star variability in the detectability of planetary phase curves splitting it into two parts. First, we applied a method based on sinusoidal signals to simulate both the stellar variability and the phase curve of an exoplanet. We assumed four different cases: WN, a light curve only composed of white noise and the phase curve itself; WNS, the same but adding a star variability of 500 ppm; WNS-F, when we try to eliminate such variability locally; and WNS-FLC if
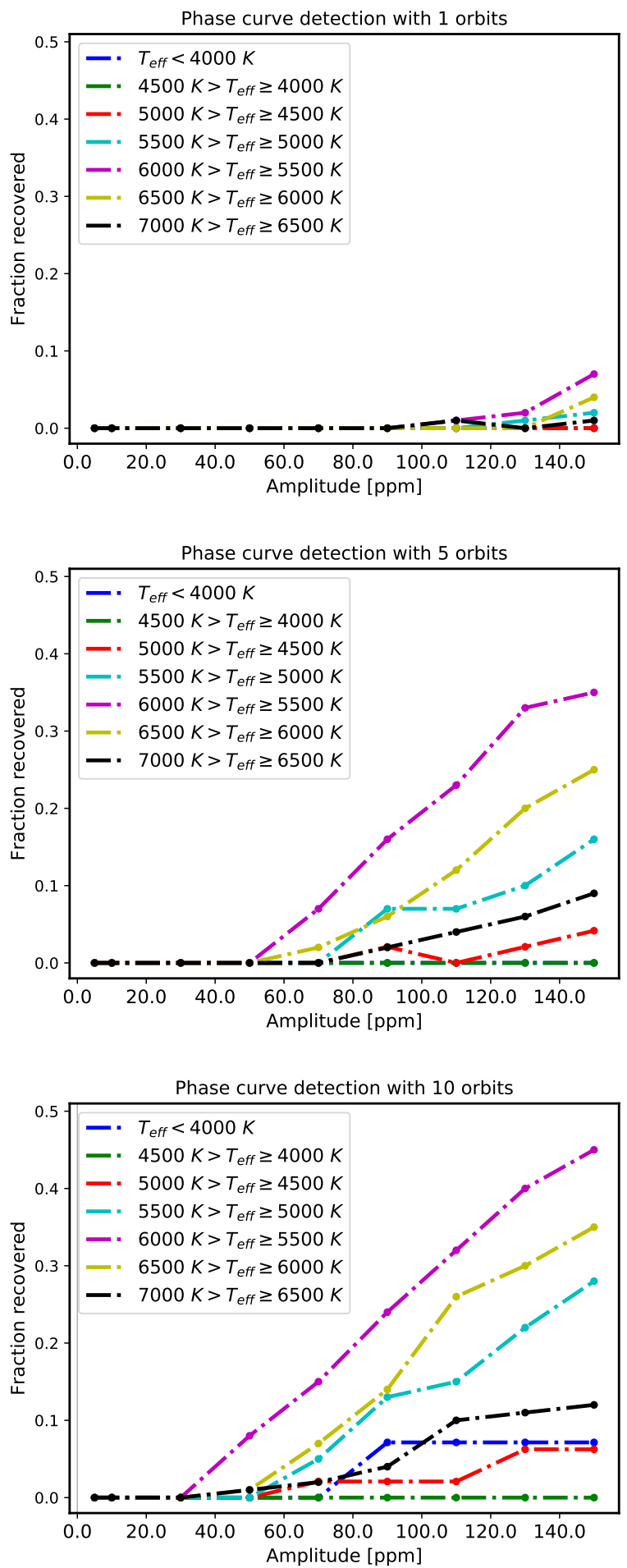

Fig. 4. Fraction of phase curves recovered as a function of the amplitude of the phase curve entered for a period of 2.5 days due to 1,5 , and 10 orbits considered (top, middle, and bottom panels, respectively). Each of the colors of the individual dotted lines represents a different sub-range of effective temperature.

we remove variability globally. Our results (see Sect. 2.4) show that we can consider that the detection of the phase curve of a planet is positive only in the most favourable cases of white noise plus a sufficiently large phase curve amplitude, taking into consideration the criterion defined in Sect. 2.3.

Second, we applied the method described above to real data from Kepler. The result shows that the stellar variability 
plays a very important role in the detectability of the phase curves. Therefore, the best detection of phase curves occurs in the effective temperature range between 5500 and $6000 \mathrm{~K}$ (Sun-like stars). In this range of effective temperature, the variability is relatively stable compared to stars of spectral type $F$ or warmer, and $K$ or cooler where variability is increased by different mechanisms that result in a smaller fraction of recovered phase curves.

For the CHEOPS telescope, where only specific events will be observed, it will take nine complete orbits to detect a phase curve with $150 \mathrm{ppm}$ of amplitude on a star with magnitude $\sim 12$ and a simple removal of variability. This is under the simple assumptions described in Sect. 2 and neglecting potential instrumental noise that can arise, and thus the number of needed orbits might be larger. One way to alleviate the problem of stellar variability in the CHEOPS data might be to intensively study the variability of the object from the ground while taking images from space with CHEOPS. These observations should cover a longer period of time and be performed on a similar bandpass to be able to fit a variability model to the star under study. This simple but costly ground-based observation time might improve the data taken from space, depending on the level of stellar variability, the amplitude of the phase curve, and the achievable precision from the ground.

The cases of TESS and PLATO are different. These two missions will take photometric measurements in an uninterrupted manner. TESS will monitor almost the whole sky, building light curves with a temporal scale of 27 days. Depending on the period, it will be possible to accumulate several orbits to better study phase curves. For PLATO, its field will be the same for a long period of time (still under study, but with a foreseen minimum of 2 years per long pointing direction), which will allow many more orbits for a deeper study of exoplanetary phase curves.

Acknowledgements. D.H. acknowledges the Spanish Ministry of Economy and Competitiveness (MINECO) for the financial support under the FPI programme BES-2015-075200, R.A. under the Ramón y Cajal program RYC-2010-06519, and the three authors under the programme RETOS ESP2014-57495-C2-R. We are grateful for the hospitality of J. Cabrera and the group of H. Rauer at the DLR Institute of Planetology, where part of this work was performed.

\section{References}

Agol, E., \& Fabrycky, D. 2017, in Handbook of Exoplanets, eds. H. J. Deeg \& J. A. Belmonte, Springer Living Reference Work, 7

Angerhausen, D., DeLarme, E., \& Morse, J. A. 2015, PASP, 127, 1113

Armstrong, D. J., de Mooij, E., Barstow, J., et al. 2016, Nat. Astron., 1, 0004

Basri, G., Walkowicz, L. M., Batalha, N., et al. 2011, AJ, 141, 20

Berdyugina, S. V. 2005, Liv. Rev. Sol. Phys., 2, 8

Borucki, W., Koch, D., Basri, G., et al. 2008, in Exoplanets: Detection, Formation and Dynamics, eds. Y.-S. Sun, S. Ferraz-Mello, \& J.-L. Zhou, IAU Symp., 249, 17

Broeg, C., Fortier, A., Ehrenreich, D., et al. 2013, Eur. Phys. J. Web Conf., 47, 03005

Brown, T. M., \& Gilliland, R. L. 1994, ARA\&A, 32, 37

Brown, T. M., Latham, D. W., Everett, M. E., \& Esquerdo, G. A. 2011, AJ, 142, 112

Cowan, N. B., \& Agol, E. 2011, ApJ, 726, 82

Demory, B.-O., de Wit, J., Lewis, N., et al. 2013, ApJ, 776, L25

Esteves, L. J., De Mooij, E. J. W., \& Jayawardhana, R. 2013, ApJ, 772, 51

Heng, K., \& Demory, B.-O. 2013, ApJ, 777, 100

Howell, S. B., Ciardi, D. R., Giampapa, M. S., et al. 2016, AJ, 151, 43

Jenkins, J. M., Caldwell, D. A., Chandrasekaran, H., et al. 2010, ApJ, 713, L120

Kitiashvili, I. N., Kosovichev, A. G., Mansour, N. N., \& Wray, A. A. 2013, ArXiv e-prints [arXiv:1312.0982]

Knutson, H. A., Charbonneau, D., Allen, L. E., et al. 2007, Nature, 447, 183

Knutson, H. A., Charbonneau, D., Cowan, N. B., et al. 2009, ApJ, 703, 769

Loeb, A., \& Gaudi, B. S. 2003, ApJ, 588, L117

López-Morales, M., \& Seager, S. 2007, ApJ, 667, L191

Mandel, K., \& Agol, E. 2002, ApJ, 580, L171

Parmentier, V., \& Crossfield, I. J. M. 2017, Astron. Nachr., 116

Rauer, H., Aerts, C., Cabrera, J., \& PLATO Team. 2016, Astron. Nachr., 337, 961

Rauer, H., Catala, C., Aerts, C., et al. 2014, Exp. Astron., 38, 249

Ricker, G. R., Winn, J. N., Vanderspek, R., et al. 2015, J. Astron. Telesc. Instrum. Syst., 1, 014003

Serrano, L. M., Barros, S. C. C., Oshagh, M., et al. 2018, A\&A, 611, A8

Shporer, A. 2017, PASP, 129, 072001

Smith, J. C., Stumpe, M. C., Van Cleve, J. E., et al. 2012, PASP, 124, 1000

Snellen, I. A. G., de Mooij, E. J. W., \& Albrecht, S. 2009, Nature, 459, 543

Stevenson, K. B., Line, M. R., Bean, J. L., et al. 2017, AJ, 153, 68

Zellem, R. T., Lewis, N. K., Knutson, H. A., et al. 2014, ApJ, 790, 53

Zucker, S., Mazeh, T., \& Alexander, T. 2007, ApJ, 670, 1326 
D. Hidalgo et al.: Role of host star variability in the detectability of planetary phase curves

\section{Appendix A: Additional plots}
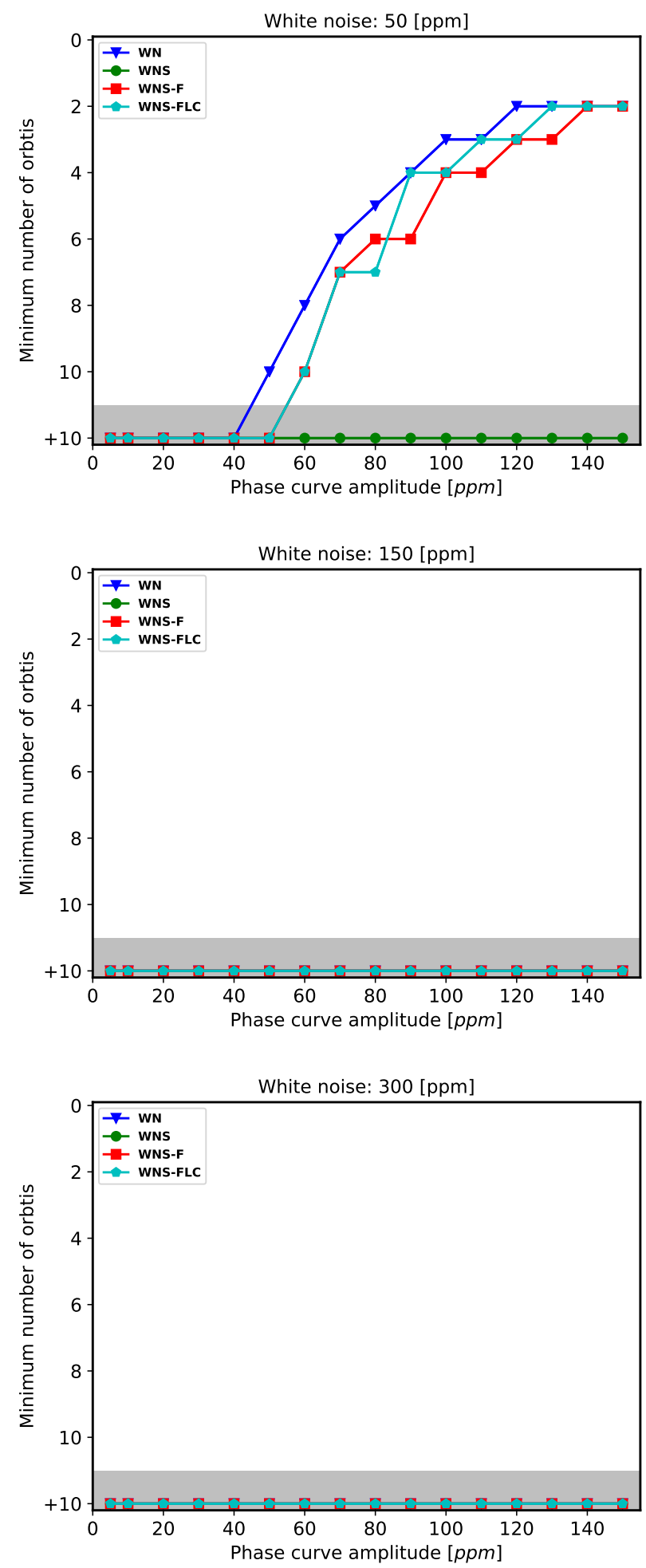

Fig. A.1. Minimum number of orbits required to have significant phase curve detection, for different white noise $(50,150$, and $300 \mathrm{ppm}$, top middle, and bottom panels, respectively) and a period of 1.0 days.
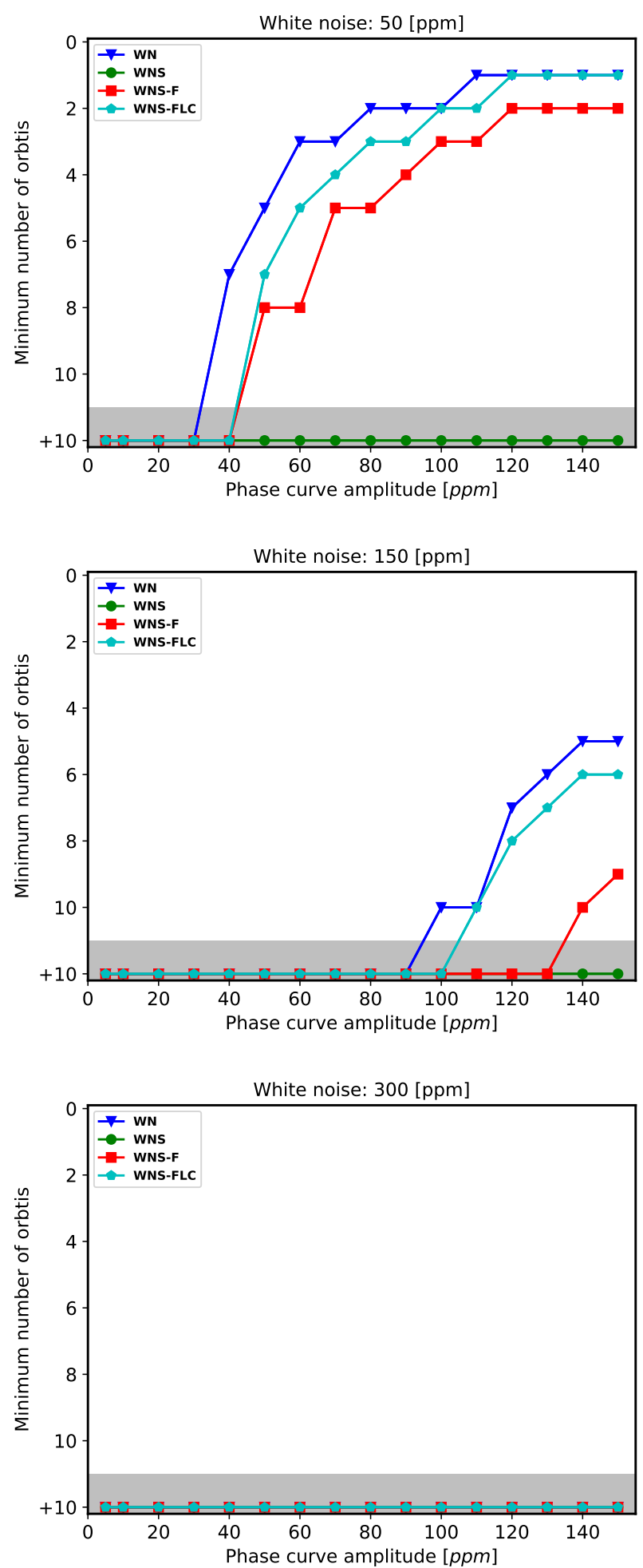

Fig. A.2. Minimum number of orbits required to have significant phase curve detection, for different white noise $(50,150$, and $300 \mathrm{ppm}$, top, middle, and bottom panels, respectively) and a period of 2.5 days. 

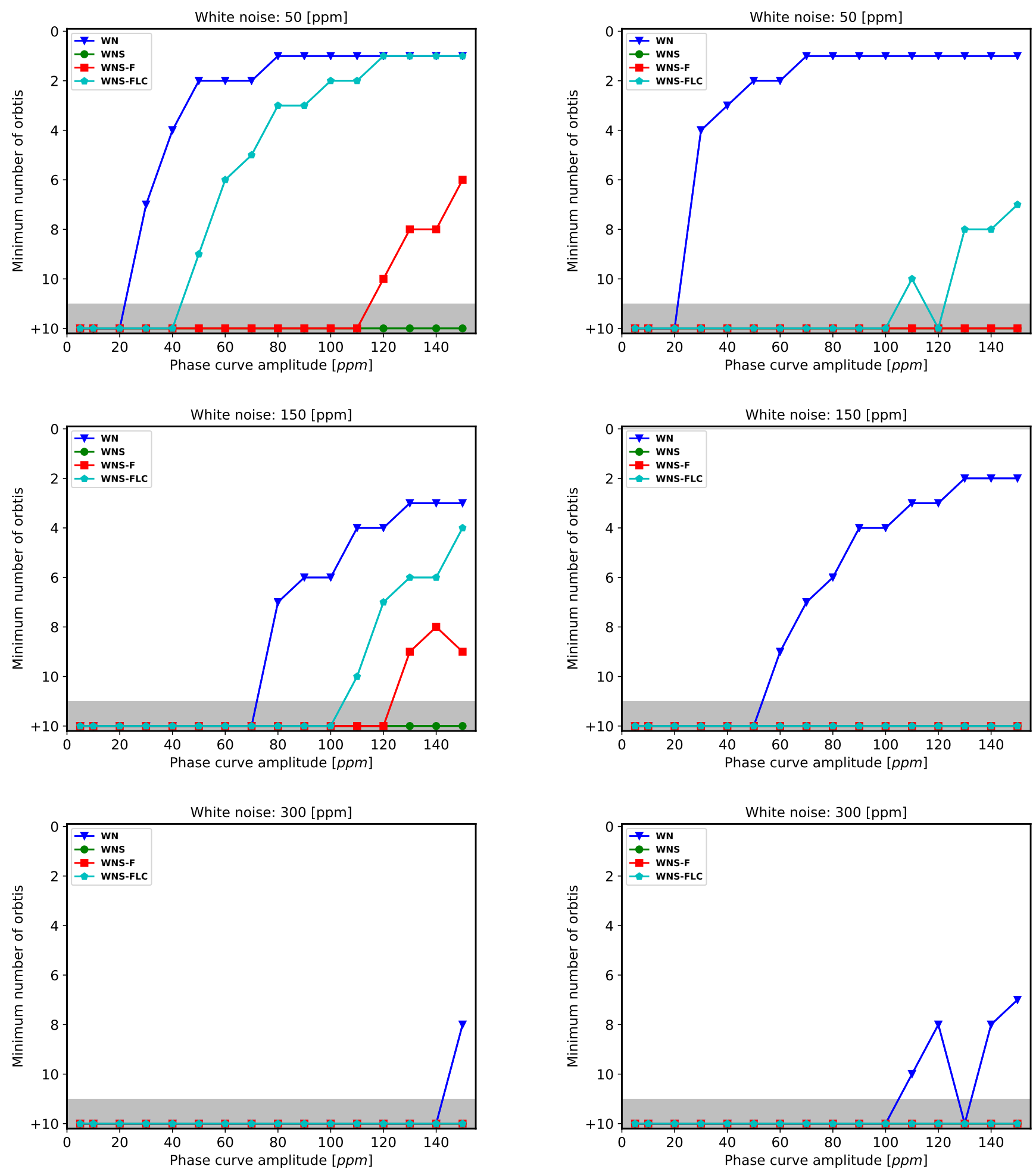

Fig. A.3. Minimum number of orbits required to have significant phase curve detection, for different white noise $(50,150$, and $300 \mathrm{ppm}$, top, middle, and bottom panels, respectively) and a period of 5.0 days.

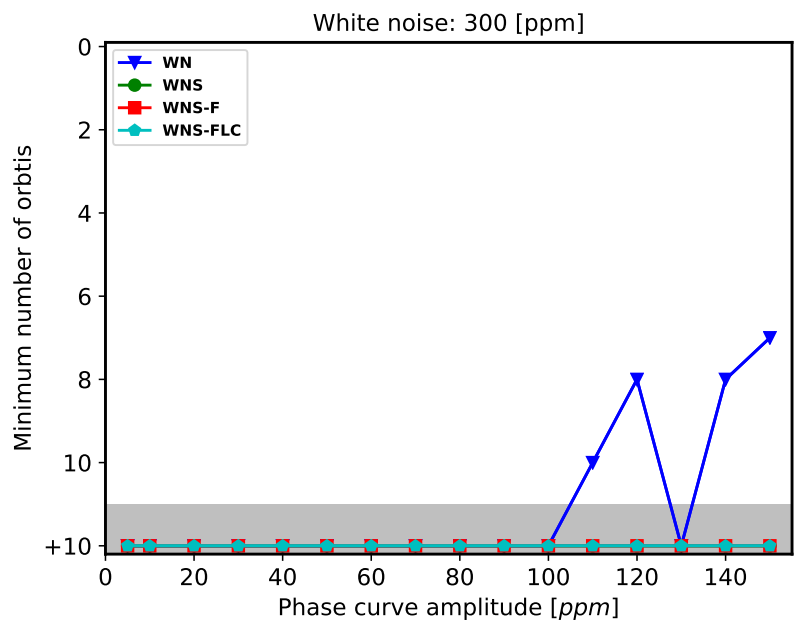

Fig. A.4. Minimum number of orbits required to have significant phase curve detection, for different white noise $(50,150$, and $300 \mathrm{ppm}$, top, middle, and bottom panels, respectively) and a period of 7.5 days. 
D. Hidalgo et al.: Role of host star variability in the detectability of planetary phase curves
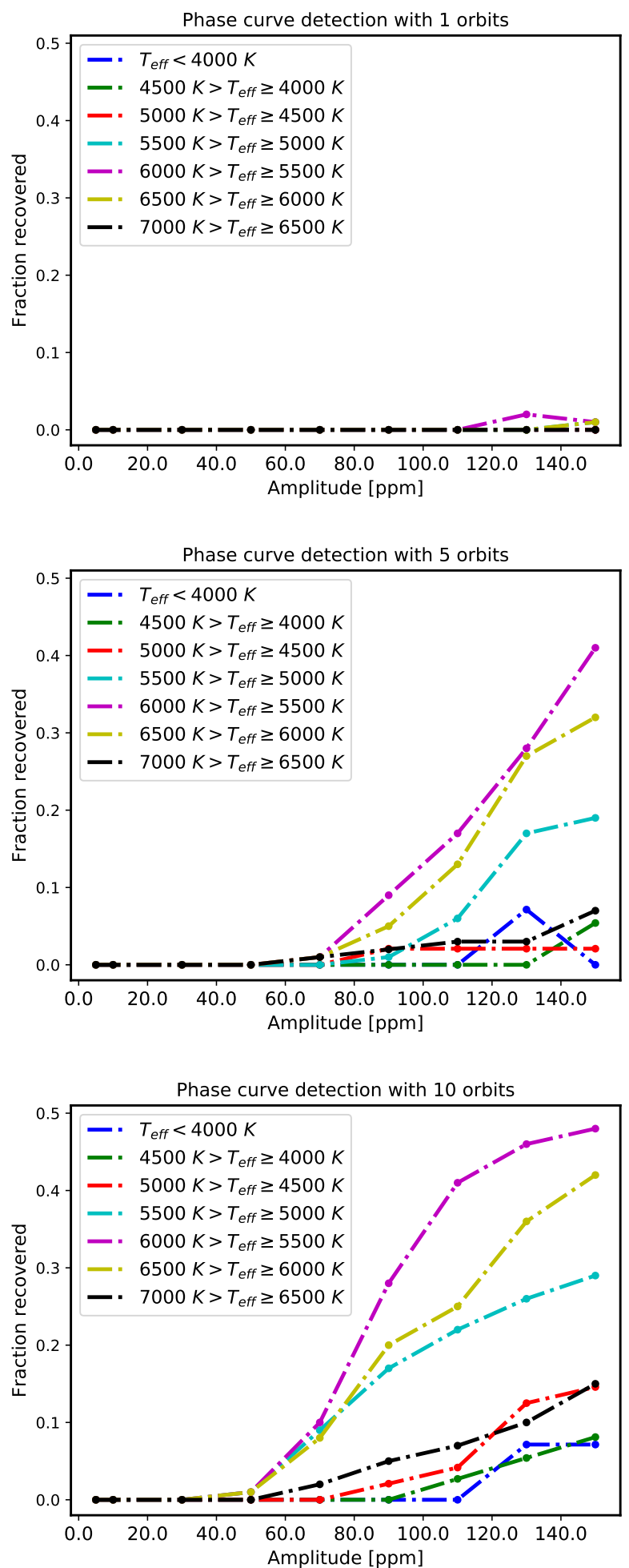

Fig. A.5. Fraction of phase curves recovered as a function of the amplitude of the phase curve entered for a period of 1.0 days due to 1,5 , and 10 orbits considered (top, middle, and bottom panels, respectively).
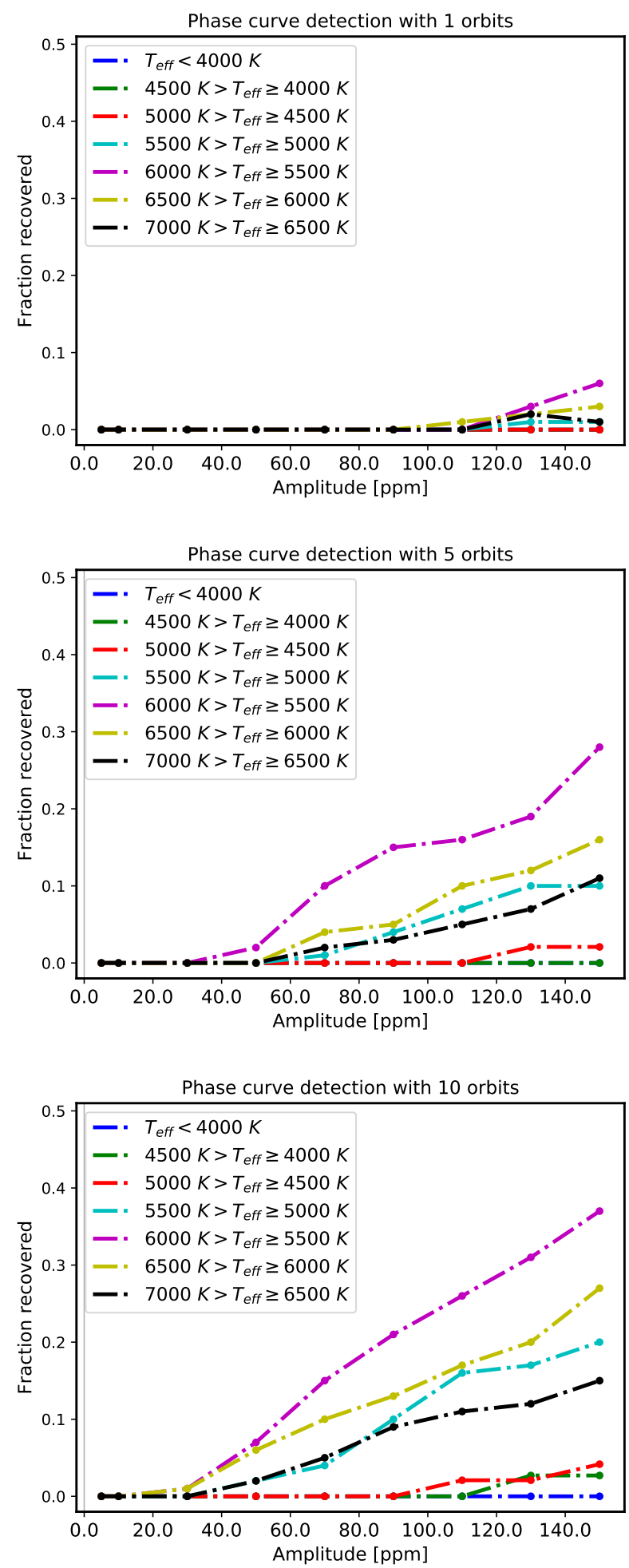

Fig. A.6. Fraction of phase curves recovered as a function of the amplitude of the phase curve entered for a period of 5.0 days due to 1,5 , and 10 orbits considered (top, middle, and bottom panels, respectively). 

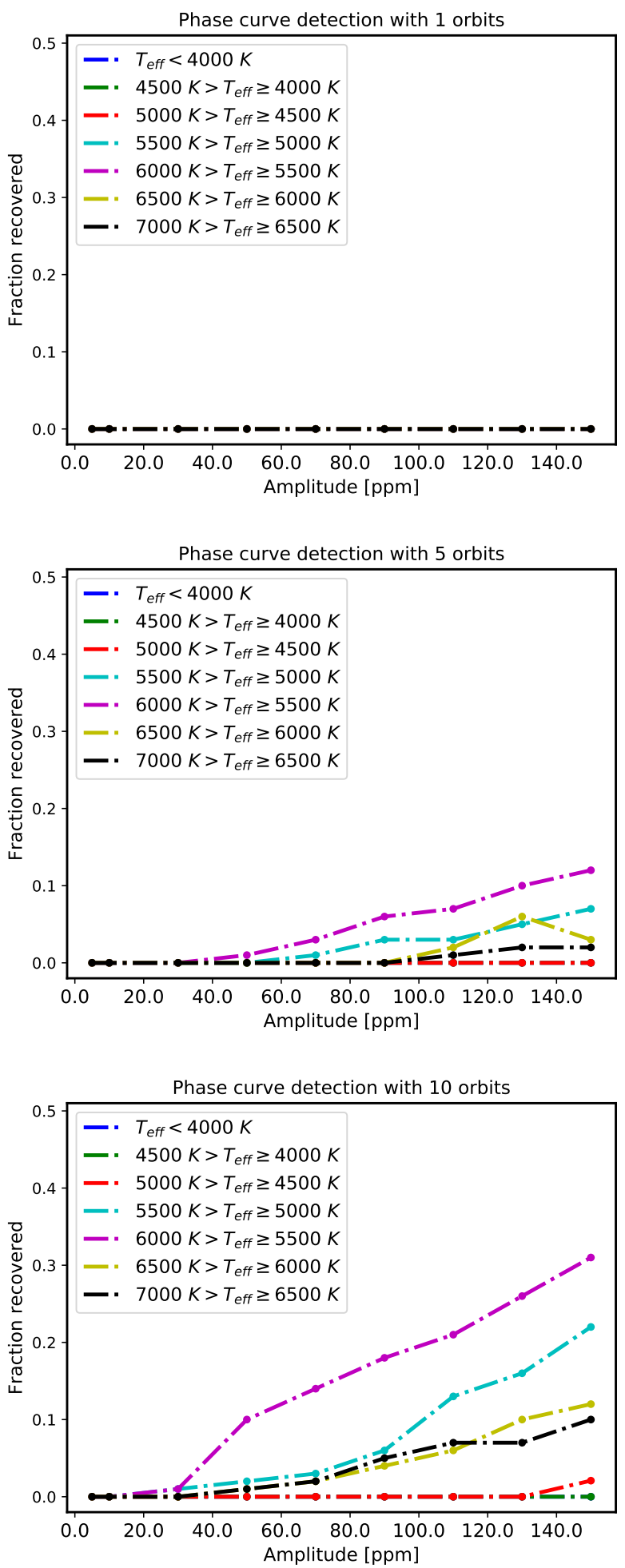

Fig. A.7. Fraction of phase curves recovered as a function of the amplitude of the phase curve entered for a period of 7.5 days due to 1,5 , and 10 orbits considered (top, middle, and bottom panels, respectively). 